\title{
Restoration of Microtia by Prosthetic Method: A Case Report
}

\author{
Mandeep Kaur, Dr. Sakshi M Kaura, Amit Sharma, Rukhsar Showkat
}

\begin{abstract}
Background: Microtia is one of the forms of ear loss and deformity. It is a congenital anomaly of the external ear. Social and psychological rehabilitation of such patients requires a prosthesis that is esthetic as well as has enough strength and texture to represent the natural ear. Because of financial constraints, some patients cannot afford the surgical procedure of grafting.

Case report: This is a case of 17 year old female with congenital missing right ear along with facial deformity due to Lower Motor Neuron facial nerve palsy.

Treatment plan: Prosthetic replacement of the missing ear was planned to improve the esthetic and social well being of the patient in an economical way.

Conclusion: a definitive management of the missing ear can be done using dental implants and bar attachment if the financial condition and social acceptance with interim prosthesis is satisfactory.
\end{abstract}

Index Terms - Congenital; Lower Motor Neuron Facial Palsy; Medical grade heat cure silicone; Microtia.

\section{INTRODUCTION}

GPT-9 describes maxillofacial prosthesis as any prosthesis used to replace part or all of any stomatognathic and/or craniofacial structures. Auricular defects can occur due to congenital malformations, trauma or surgical removal of neoplasms [1].

Microtia is considered the most common congenital defect with incidence rate of 1 in 12,500 live births. It is usually associated with atresia of the external auditory canal. The classic remnant usually seen in microtia is commashaped, with the upper portion containing a small nubbin of deformed cartilage. Complete absence of the auricle or anotia is extremely rare. Microtia and anotia are part of a spectrum of first and second branchial arch abnormalities known as hemifacial microsomia. They are associated with ear anomalies and preauricular tags [1].

Microtia has been classified by various authors but the most accepted classification was proposed by Marx and described by Rogers [2]:

Published on July 22, 2020

Mandeep Kaur, Department of Prosthodontics, Luxmi Bai Institute of Health and Dental Sciences, Patiala.

(corresponding e-mail: dr.mandeepkaur ${ }^{@}$ ymail.com)

Sakshi M Kaura, Department of Prosthodontics, Luxmi Bai Institute of Health and Dental Sciences, Patiala.

(e-mail: sakshikaura@gmail.com)

Amit Sharma, Department of Prosthodontics, Luxmi Bai Institute of Health and Dental Sciences, Patiala.

(e-mail: amit_sharma2035@yahoo.com)

Rukhsar Showkat, Department of Prosthodontics, Luxmi Bai Institute of Health and Dental Sciences, Patiala.

(e-mail: rukhsarshowkat2611@gmail.com)
Grade I: The auricle is small and the different structures of each part of the ear are recognizable.

Grade II: The ear is smaller than normal, and the auricular remnant retains some structural resemblance to a helix in its configuration.

Grade III: The auricular configuration is absent.

Cosmetic surgical correction is one of the therapeutic approaches but it involves additional surgical procedure [3]. Financial condition and underlying medical conditions may limit the use of this approach. Prosthetic fabrication of the missing part using medical grade silicone is the alternative method of correction of the malformation. Prosthetic reconstruction is simple, less time consuming, cost effective with less morbidity as compared to cosmetic surgical reconstruction [4].

Fabrication of an extra-oral prosthesis is considered as an artist work as the form, color and texture must closely match and camouflage the surrounding natural tissues. The various treatment options available include mechanically retained prosthesis, bio-adhesive retained prosthesis, implant retained and the recently developed rapid prototyping and Computer Aided Designing - Computer Aided Machining (CADCAM) developed prosthesis [4].The prosthetic ear can retain eyeglasses and a hearing aid if needed [5].

\section{CASE REPORT}

If A 17-year-old female patient reported to the Department of Prosthodontics for the replacement of missing right ear. On clinical examination, a tissue tag of the remnant of right ear was seen with some facial distortion (Fig. 1).

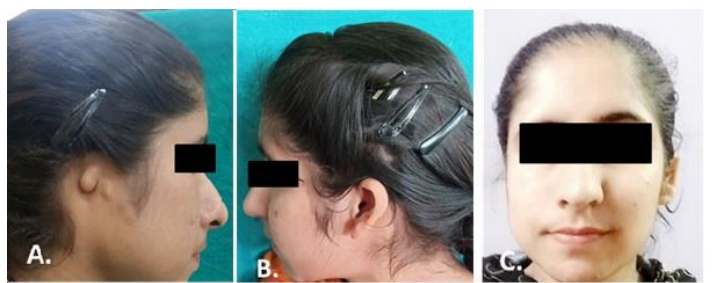

Fig.1. Pre-operative A.- Right profile, B. - Left profile, C- Front profile.

History revealed the presence of microtia (Grade-III) with respect to right ear with Lower Motor Neuron facial palsy of the right side. CT scan and MRI revealed ankylosis of the stapes of middle ear resulting in some hearing loss. Parents of the patient refused any surgical procedure because of the financial limitations. They were given the option of adhesive retained prosthesis to which they agreed.

Impressions of both the ears were made with the help of polyvinyl siloxane putty material (Fig. 2). 


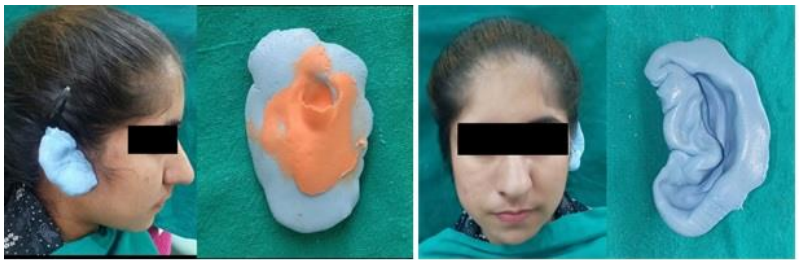

Fig.- 2: Impressions made with polyvinyl siloxane

Casts were poured in type IV gypsum stone. Carving and sculpting of the ear was done with the help of modeling wax (Fig. 3).

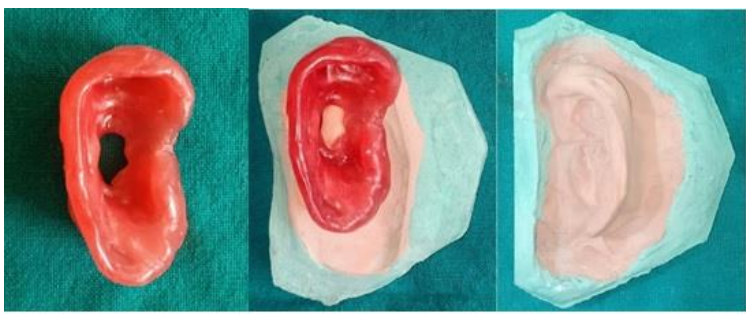

Fig. 3. Wax trial made

Try-in of the prepared wax ear was done (Fig. 4). Because of the asymmetry of the face, there was a difference in visibility of the pinna of the ear. Esthetics was verified by the patient and her parents. After approval of the shape of the ear, shade selection was done.

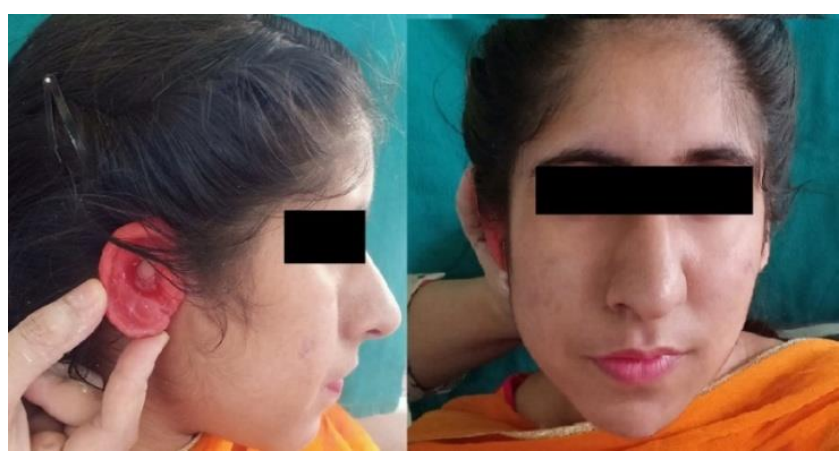

Fig. 4. Try-in done

Small amount of heat cured medical grade silicone was mixed and colors were added to match the skin tone. The mixed shade was placed on a clear cellophane sheet and then placed over the face of the patient to verify the shade. The shade was made darker or lighter until it completely camouflages the adjoining skin (Fig. 5). The shade was verified by the patient and the final cellophane sheet was kept as reference.
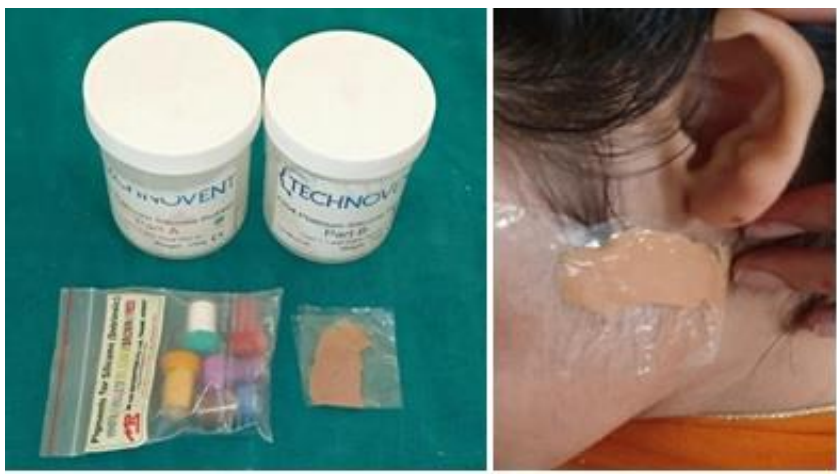

Fig. 5. Shade selection done
The flasking process was started after approval of shape and shade from the patient. For accommodation of sunglasses and as an additional retentive aid, a thin wire loop was placed on the supero-lateral aspect of the wax pattern. Flexible denture flask was used for flasking. After completion of the flasking, wax elimination was done (Fig. $6)$.

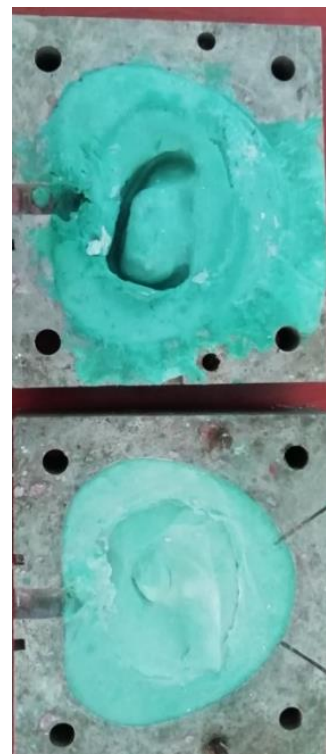

Fig. 6. Separating medium applied after dewaxing process

The flasks thus obtained had channel for pouring of the silicone impression material. A thin layer of separating medium was applied and allowed to set (Fig. 7).

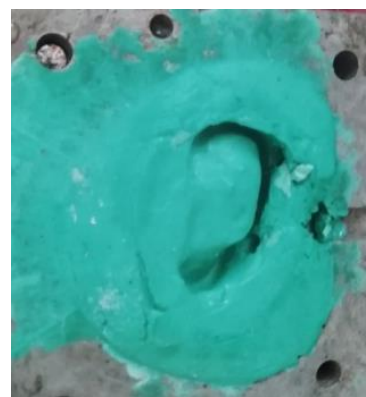

Fig. 7. Channels in flexible flask for flow of material

Medical grade heat temperature vulcanized (HTV) silicone (Technovent Z004) was mixed with appropriate intrinsic colors (MP Sai) (Fig. 8).

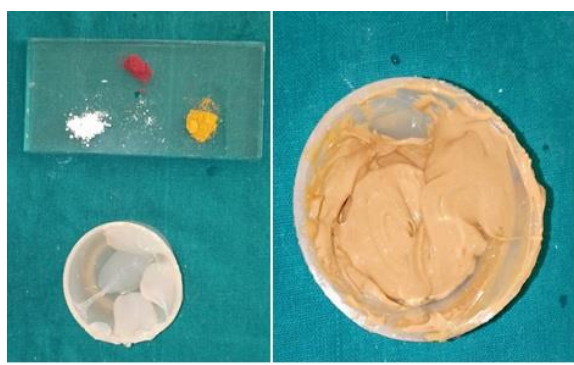

Fig. 8. HTV silicone mixed to appropriate shade

After the desired shade was obtained, the mixed material was poured into flask with the help of $10 \mathrm{ml}$ syringe to reduce air entrapment and obtain uniform texture and color.

The flask was then placed in water for 1.5 hours as per manufacturer's recommendations. After completion of 
flasking cycle, the flask was bench cooled for 1 hour. The prosthesis was retrieved from the flask (Fig. 9).

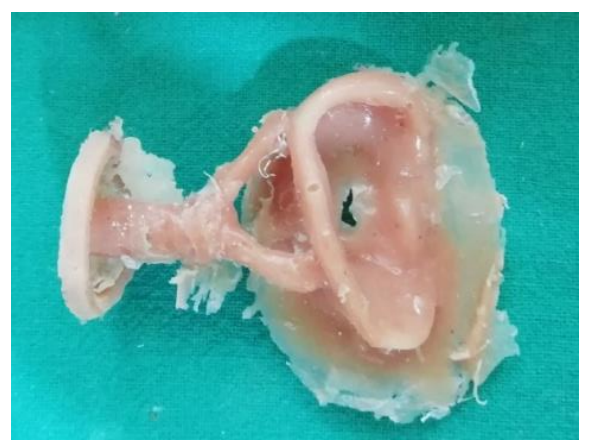

Fig. 9. Retrieved prosthesis with sprues

After trimming the sprues, the finishing and polishing of the prosthesis was done with silicone polishing burs (Fig. $10)$.

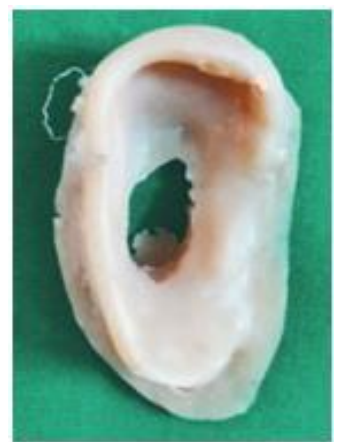

Fig. 10. Final prosthesis

Final prosthesis was retained with the help of adhesive (Fig. 11).

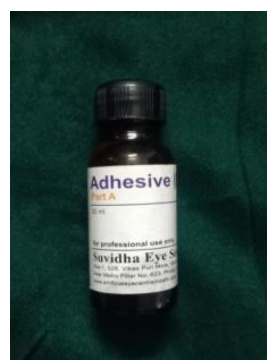

Fig. 11. Adhesive

Final prosthesis resembled the natural counterpart (Fig. 12).

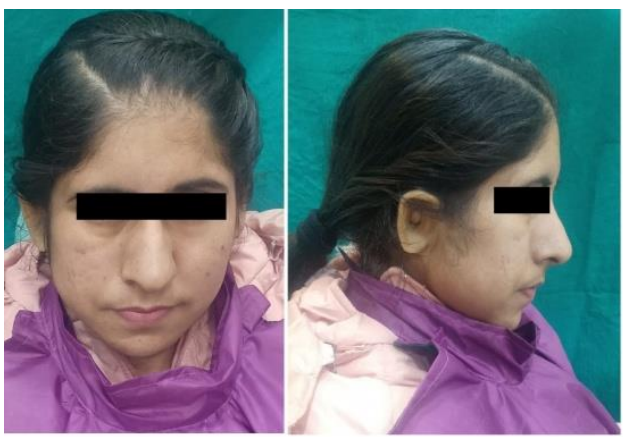

Fig. 12. Post-operative
Patient was explained about the limitations of the prosthesis and daily care of the prosthesis. Patient and parents of the patient were satisfied with the esthetics of the prosthesis.

\section{DISCUSSION}

In Congenital deformity, the ear does not fully develop during first trimester of pregnancy whereas acquired causes could be burns, trauma, or malignancies (most common malignancies include basal cell carcinoma, squamous cell carcinoma and malignant melanoma). Aim of maxillofacial rehabilitation is to provide a suitable prosthesis for patient with facial deformity to restore the esthetics and make them socially acceptable. Surgical rehabilitation of auricular defects can be done with autogenous tissues but may not be feasible for personal or medical reasons [4]. So, a good alternative is to develop an auricular prosthesis.

Fabrication of unilateral auricular prosthesis is more difficult as compared to bilateral auricular prostheses, as it presents a constant comparison with a natural counterpart. There are different methods for sculpting an auricular prosthesis-

- Obtaining the reverse image of the ear of a sibling with close resemblance to that of patient's ear or any other person with compatible ear morphology can be used (Donor technique).

- Mirror image cast of the patient's remaining ear, or more ideally obtaining a presurgical cast.

In 1980, Nusinov and Gay described a method for obtaining the reverse image of an ear by using parallel lines transferred to casts, a vertical camera capable of reproducing three dimensional objects, and tracing paper [6]. Shimodaira et al proposed superimposing a color slide onto a facial cast to sculpt a facial prosthesis [7]. Both methods are relatively complex and require special and costly equipment.

In this case, measurement of the patient's normal ear was done using calipers. The entire length of the ear, the antero posterior dimension, and the height of elevation of the pinna from the skin beneath the dorsal surface of the ear were measured. The shape and pattern of the helix, antihelix, concha and lobule of the normal contralateral ear was also carefully observed and detailed photography was carried out. Using these as references, free hand carving of the modelling wax was done over the cast of the defected ear to make the wax pattern. Due to presence of anatomical deviation from the normal of the ear which did not resemble to any close relative or parents, the donor technique could not be used in this case.

Heat Temperature Vulcanized (HTV) Medical Grade Silicone was used. Silicone is the material of choice for facial prosthesis because of its flexibility and life like appearance. Intrinsic stains were used for coloration as they are more stable and provide better aesthetic results [8]. With time intrinsic stains undergo less color alteration as compared to extrinsic coloration methods. HTV silicone was used to overcome the disadvantages of hardening of prosthesis and slight discoloration with time due to extreme climatic conditions like hot, humid, sweat, dust, pollens and other offenders in Room Temperature Vulcanized silicones. 
Special flexible denture flasks were used in this case so as to avoid the entrapment of air while packing of the silicone material as the material has comparatively less viscosity. Pressure from the syringe used made sure that the adequate material flowed to all the undercut areas and even the thinnest portion of the pinna.

Retention is the most important factor in the success of a prosthesis. Implant retained prosthesis ought to be given prime consideration owing to its improved retention, stability and comfort for the patient [5], however, due to financial constraint the same was overlooked in the instant case. Prosthesis was fitted with the help of anatomical undercuts available from the remnants of auricle on right side and also from a bio-adhesive, which provides better retention for a longer period of time as it doesn't get dissolved on contact with sweat.

Nowadays new techniques for the fabrication of ear prosthesis have come up and include Rapid prototyping technology [9], Computed Tomography scanner and 3Dimensional systems [10]. Although costly, they facilitate the production of mirror image of auricular prosthesis having high accuracy and overcome the limitations of conventional prosthetic techniques. Tissue engineering and stem cell tissue regenerations have also resulted in the formation of new tissue which will help the prosthodontic regeneration in future.

\section{CONCLUSION}

This article describes the method of fabrication of prosthetic ear using the flexible denture flasks with the help of syringe to minimize the intrinsic as well as extrinsic porosities. The pressure packing of the heat cure medical grade silicone removes any air bubbles that can be incorporated during the packing procedure. The heat cure medical grade silicone was used as it has an advantage of better esthetics having life like appearance, good edge strength, longer working times and longer duration of color sustainability as compared to room temperature medical grade silicone. Patient was satisfied with the esthetics and the ease of use and maintenance of the prosthesis. On her ollow up visit, she was happy with the social acceptance of the prosthesis which overcame her psychological fears. Spectacle retention loop aids in retention as well as esthetics. Adhesive was used as the primary retentive agent.

\section{REFERENCES}

[1] Beumer J, Curtis TA and Marunick MT. Maxillofacial rehabilitation Prosthodontic and Surgical Considerations.1st edn.Ishiyaku Euroamerica;1996, ch-9, pp. 377-402

[2] Weerda H, "Classification of congenital deformities of the auricle," Facial Plast Surg Aesthet Med., vol.5, pp. 385-388, October 1988.

[3] Mardani MA, Aminian G, Tabatabaian F, Arazpour M, Hutchins SW and Head JS, "A novel technique for fabricating an ear prosthesis in a patient with congenital ear deformity," ProsthetOrthot Int., vol. 37,pp. 340-343, August 2013.

[4] Singh A, Ghosh S, Kar S and Ahmed I, "Silicone prosthesis for a patient with unilateral ear defect: A clinical case report," European $J$ Gen Dent., vol. 2, pp. 315-319, 2013.

[5] Chinnasamy A, Gopinath V and Jain AR, "Ear Prosthesis for Postburn Deformity," Case Rep Otolaryngol, April 2018.
[6] Nusinov NS, Gay WD, "A method for obtaining the reverse image of an ear," J Prosthet Dent., vol. 44, pp. 68-71, July 1980.

[7] Shimodaira K, Fukuda H, Kanazawa T, Funakubo T, "Technique for superimposing a color slide onto a facial cast to sculpt a facial prosthesis," J Prosthet Dent., vol. 62, pp. 212-213, August 1989.

[8] Taylor,T.D, Clinical Maxillofacial prosthetics, Chicago. Quintessence Publ Co, 2000,ch-16, pp. 233-244

[9] Al Mardini M, Ercoli C, Graser GN, "A technique to produce a mirror- image wax pattern of an ear using rapid prototyping technology,"J Prosthet Dent, vol. 94, pp. 195-198, August 2005.

[10] Jiao T, Zhang F, Huang X, Wang C, "Design and fabrication of auricular prostheses by CAD/CAM system," Int J Prosthodont, vol. 1, Issue 4, pp. 460-463, July/August 2004.

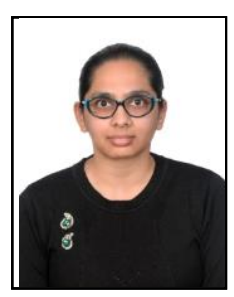

Mandeep Kaur* (Corresponding Author)

Born in Punjab 1989, Dr. Mandeep Kaur graduated from Baba Farid University of Health Sciences in 2012 and did Masters in Prosthodontics and Crown \& Bridge from the Baba Farid University of Health Sciences in 2017.

She is working as a Senior Lecturer in department of Prosthodontics and Crown \& Bridge in Baba Farid University of Health Sciences since 2017. She is also working as a Prosthodontist at Apex Dental Care since 2017. Among the publications:

Kaur M, Kumar S, Kaura SM, Kaur S, Singh J, Fabrication of flangeless complete denture to improve esthetics: a case report, international journal of clinical and diagnostic research, 2018;6,2,1-8. 